\title{
Distinct Microbiome in Pouchitis Compared to Healthy Pouches in Ulcerative Colitis and Familial Adenomatous Polyposis
}

Garrett C. Zella, MD, Elizabeth J. Hait, MD, MPH, Tiffany Glavan, BA, Dirk Gevers, PhD, Doyle V. Ward, PhD, Christopher L. Kitts, PhD, and Joshua R. Korzenik, MD

Background: Pouchitis occurs in up to 50\% of patients with ulcerative colitis (UC) undergoing Heal pouch anal anastomosis (IPAA). Pouchitis rarely occurs in patients with familial adenomatous polyposis (FAP) who undergo IPAA. Our aim was to compare mucosal and luminal flora in patients with UC-associated pouchitis (UCP), healthy UC pouches (HUC), and healthy FAP pouches (FAP).

Methods: Nineteen patients were enrolled in this cross-sectional study (nine UCP, three HUC, seven FAP). Patients with active pouchitis were identified using the Pouchitis Disease Activity Index (PDAI). Heal pouch mucosal biopsies and fecal samples were analyzed with a 16S rDNA-based terminal restriction fragment length polymorphism (TRFLP) approach. Pooled fecal DNA from four UCP and four FAP pouches were sequenced for further speciation.

Results: TRFLP data revealed statistically significant differences in the mucosal and fecal microbiota between each group of patients. UCP samples exhibited significantly more TRFLP peaks matching Clostridium and Eubacterium genera compared to HUC and FAP pouches and fewer peaks matching Lactobacillus and Streptococcus genera compared to FAP. DNA Sanger sequencing of a subset of luminal samples revealed UCP having more identifiable sequences of Firmicutes (51.2\% versus 21.2\%) and Verrucomicrobia (20,2\% versus 3,2\%), and fewer Bacteroidetes (17,9\% versus 60.5\%) and Proteobacteria (9,8\% versus $14,7 \%$ ) compared to FAP. 
Conclusions: The pouch microbial environment appears to be distinctly different in the settings of UC pouchitis, healthy UC, and FAP. These findings suggest that a dysbiosis may exist in pouchitis which may be central to understanding the disease.

Abdominal colectomy with ileal pouch anal anastomosis .(IPAA) is the preferred surgery for patients with ulcerative colitis (UC) unresponsive to medical therapy or with dysplasia and for those with familial adenomatous polyposis syndrome (FAP), ${ }^{\mathrm{I}}$ Inflammation of the ileal pouch, termed pouchitis, is the most common long-term complication in patients with UC who undergo IPAA. ${ }^{2}$ Pouchitis occurs in up to $50 \%$ of these patients ${ }^{3 / 4}$ and is the major factor leading to morbidity after IPAA surgery in patients with UC. ${ }^{3, t}$ In comparison to individuals with a history of UC, those undergoing IPAA for FAP are far less likely to develop pouchitis. ${ }^{5}$

The pathogenesis of pouchitis is incompletely understood. Proposed etiologies include fecal stasis, bacterial overgrowth, dysbiosis, genetic susceptibility, short-chain fatty acid deficiency, ischemic complications of surgery, bile-acid toxicity, immune alteration, a missed diagnosis of Crohn's disease (CD), or a recurrence of UC in the pouch. ${ }^{1.6,1}$ Pouch bacteria appear central to the pathophysiology of the disease. The efficacy of antibiotics in ameliorating pouchitis underscores the role of mucosal or luminal bacteria in the development of pouchitis." The role of gut microbes in pouchitis is further supported by studies revealing fewer pouchitis relapses and postoperative prophylactic benefit with probiotic therapy..$^{\circ}$ A therapeutic utility of both antibiotics and probiotics suggests there may be subtle shifts in pouch microflora that culminate in pouch inflammation.

Recently, Komanduri et al " described a pouch dysbiosis in five patients with UC and pouchitis when compared to patients with UC and no pouchitis and to healthy ileal tissue in continuity with the colon. Inflamed pouch mucosa was found to have greater bacterial species diversity than pouch 
control or healthy ileal tissue. Fusobacterium varium was more abundant in pouchitis tissue, while Streptococcus species were dramatically reduced. Healthy UC pouches possessed a unique floral pattern, with more representatives from the Clostridium, Enteric, and Streptococcus groups compared to ileal tissue from healthy subjects.

In our study we aimed to characterize both mucosal and luminal microflora in inflamed UC pouches compared to healthy UC and FAP pouches. We designed the study with FAP pouch control subjects in an effort to find shifts in flora that were distinct to UC and inflammation rather than fecal stasis. We utilized 16S ribosomal DNA gene-based terminal restriction fragment length polymorphism (TRFLP) techniques to characterize luminal and mucosal microbial environments, followed by DNA sequencing for further characterization and validation.

\section{MATERIALS AND METHODS}

\section{Patient Population}

Individuals over age 18 years were enrolled from the Massachusetts General Hospital (MGH) Gastrointestinal Unit in one of three cohorts with pouches after IPAA: those with a history of UC and subsequent pouchitis (UC pouchitis, UCP), those with a history of UC without pouchitis (healthy UC pouch, HUC), or those with a history of FAP without pouchitis (healthy FAP pouch, FAP). The diagnosis of UC and FAP in these patients was based on standard clinical, radiologic, and histologic diagnostic criteria. UCP patients were eligible if they had experienced at least two episodes of antibiotic-responsive pouchitis symptoms in the previous 2 years or had persistent pouchitis symptoms despite two courses of antimicrobial therapy. A Pouch Disease Activity Index (PDAI, comprising clinical, endoscopic, and histologic elements) score $>7$ was required for inclusion in the UCP group. ${ }^{2}$ Eligible UCP patients underwent colectomy with IPAA more than 3 months prior to enrollment. Eligible HUC and FAP patients underwent colectomy with IPAA more 
than 1 year prior to enrollment without experiencing pouchitis symptoms. Exclusion criteria for each group included any indication of CD and the use of probiotics, biologic or other immunosuppressive therapy, rectal therapy, or new antibiotics during the previous 2 months. Chronic antibiotic use for more than 3 months was permitted in the UCP group if the patient's symptoms persisted without the use of new antibiotics.

\section{Data Collection}

Key clinical data collected from each patient included age, gender, disease duration and extent, pouch duration, number of pouchitis episodes in the previous year, medication use, and the clinical components of the PDAI. The PDAI clinical score takes into account stooling frequency and the presence of hematochezia, stooling urgency, abdominal cramping, and fever. Endoscopic and histologic findings were noted for each patient and incorporated into the PDAI score.

\section{Specimen Collection}

All patients underwent pouchoscopy after clinical data were collected. Patients received a short course of bowel preparation 1 day prior to the procedure with phospho-soda. Luminal pouch contents were suctioned through the colonoscope into a sterile container. Approximately I cc of the suctioned stool was aliquoted into each of three vials containing 1 cc of $10 \%$ glycero $1 / 90 \%$ sterile water. Tubes were inverted to generate a homogeneous mixture then immediately placed in dry ice. The pouch was examined for elements of the PDAI endoscopic score: edema, granularity, friability, loss of vascular pattern, mucous exudates, and ulceration. Then three biopsies were taken at the most inflamed sites or at random locations from normal-appearing tissue. Each tissue specimen was immediately rinsed in normal saline then placed in a vial containing 1 cc of $10 \%$ glycerol $/ 90 \%$ sterile water. The vials were inverted to submerge the tissue 
specimen then immediately placed in dry ice. All specimens were then stored at $-80^{\circ} \mathrm{C}$ within 30 minutes.

\section{TRFLP Analysis}

All stool and tissue specimens were analyzed by TRFLP at the Environmental Biotechnology Institute at California Polytechnic State University (CPSU). DNA was isolated using MoBio's Power Soil DNA extraction kit according to the manufacturer's protocol (MoBio Laboratories, Carlsbad, CA). Triplicate 0.1-g samples were used for the extraction of stool samples, while the entire biopsy sample was used for the isolation of DNA from tissue samples.

Bacterial 16S ribosomal RNA genes were amplified from both types of sample using the forward primer 8DF (5'-AGA GTT TGT TCM TGG CTC AG-3') and the reverse primer 536K2R (5'-GTA TTA CCG CGG CTG CTG G-3'). The forward primer was fluorescently labeled with a phosphamide dye. Fifty microliter reactions were assembled using $1_{\mathrm{FL}}$ of undiluted DNA, 5 pl., of 10x buffer, 3 pl. of 10 mM dNTPs, 2 tL of 20 mg/mL BSA, 7 pL of 25 mM $\mathrm{MgCl}_{2}, 1 \mathrm{tL}$ of each primer, and 0.3 ,td, of $5 \mathrm{U} / \mathrm{L}$ TaqGold (Applied Biosystems, Foster City, CA).

Reaction temperatures and times were as follows: $95^{\circ} \mathrm{C}$ for 10 minutes followed by 30 cycles of $95^{\circ} \mathrm{C}$ for 1 minute, $60^{\circ} \mathrm{C}$ for 1 minute, and $72^{\circ} \mathrm{C}$ for 2 minutes, followed by a final extension of $72^{\circ} \mathrm{C}$ for 10 minutes. The quality of the extractions and polymerase chain reaction (PCR) reactions were confirmed using gel electrophoresis. PCR triplicates were combined during a PCR cleanup performed using MoBio's PCR Cleanup Kit following the manufacturer's protocol (MoBio Laboratories). PCR products were quantified using a FLX800 microplate fluorescence reader tuned to the labeling dye (Bio-Tek Instruments, Winooski, VT). 
Three enzyme digests were independently performed using cleaned-up PCR product and the restriction endonucleases HaeIH, Hpall, and Alul (New England Biolabs, Beverly, MA). Each 40-1,tL digest included 75 ng DNA, 1 U enzyme, and 4 pi, buffer. Samples were digested for 4 hours at $37^{\circ} \mathrm{C}$ and the enzyme was inactivated for 20 minutes at either $65^{\circ} \mathrm{C}(\mathrm{HpaII}$ and Alul) or $80^{\circ} \mathrm{C}$ (Haell1). Resulting fragments were ethanol precipitated and resuspended in 20 [IL formamide and 0.25 tL CEQ 600 basepair standard. Terminal restriction fragments were separated using capillary gel electrophoresis and profiles were obtained using a Beckman Coulter (Fullerton, CA) CEQ8000X DNA analysis system.

Terminal restriction fragment lengths and relative peak areas were exported from the CEQ8000 into Excel (Microsoft, Seattle, WA). TRF data consists of peak sizes, which indicate the length of the DNA fragment in nucleotides, and the area under each peak, which provide a measure of abundance for each DNA fragment. Since the amount of DNA loaded on the capillary cannot be accurately controlled, the sum of the total peak area varies between TRF patterns. Therefore, peak areas are normalized by converting the value to parts per million to standardize the data for comparison." Peaks with an area less than 10,000 ppm ( $<1.0 \%$ of the total) were excluded from the analysis to reduce excess noise. Data from three independent restriction enzyme digests were included to reduce the incidence of distinct sequences with equivalent TRF lengths. TRF fragments determined to differ most between sample groups were compared to available GenBank (Bethesda, MD) sequences to tentatively identify potential bacterial populations. TRFLP data were analyzed for peaks that could be identified by database analysis as representing particular groups of organisms.

\section{S Ribosomal DNA-based DNA Sequencing}

Four fecal samples from each of the UCP group and the FAP group were selected for sequencing analysis of pooled DNA. Samples with the greatest intersample similarities in TRF 
profiles (as determined with Bray-Curtis similarity indices) were selected in an effort to create the most uniform pooled specimens representative of each patient group. DNA from the samples was processed as detailed for TRF analysis, using full-length 16S PCR primers without fluorescent label, then sent to the Broad Institute (Cambridge, MA) for sequencing. Samples were cloned into the pCR2.1-TOPO vector and sequenced on an ABI3730 DNA sequencer.

Sequences were trimmed using LUCY. ${ }^{14}$ Read pairs from each clone were then assembled using the following alignment-assisted assembly method implemented at the Broad Institute. A reference sequence for use as an alignment template for the read pairs was first selected from a core set of nonchimeric 16S rRNA sequences obtained from Greengenes. ${ }^{15}$ The core set reference sequence sharing the greatest number of matching k-mers with the aggregate k-mer set of both forward and reverse reads was selected. Forward and reverse reads were each aligned to the core reference sequence using Blast. ${ }^{16}$ Aligned forward and reverse reads were then assembled based on the alignment with base quality scores used and preserved through assembly.

For purposes of classification, only assembled sequences of greater than 1100 nucleotides were considered. Sequences were classified using a naïve Bayesian rRNA classifier." The classifier was trained on the Ribosomal Database Project core set, RDP10. ${ }^{18}$

\section{Statistics}

Differences in patient characteristics were assessed using Fisher's Exact test for categorical variables and the Exact Kruskal—Wallis test for continuous variables. TRFLP datasets were transformed by taking the square root of the area under each peak to deemphasize large TRFLP peaks while still accounting for relative abundance. Transformed data from UCP, HUC, and FAP groups were compared using Bray-Curtis similarity, multidimensional scaling, and analysis of similarity (ANOSIM) (Primer E, Plymouth, UK). ANOSIM was also used to compare 
intersubject and intrasubject TRFLP profile similarities. Analysis of variance (ANOVA) was used to compare relative abundance of specific TRFLP pattern elements between each group of subjects. Among the pooled fecal DNA samples, differences in the percentage of total identifiable clones represented by each bacterial genus were calculated using Fisher's Exact test, considering that the number of identifiable clones in a sample is proportional to species abundance.

\section{Ethical Considerations}

This study was approved by the Institutional Review Boards at MGH and CPSU.

[Insert Table 1]

\section{RESULTS}

\section{Patients}

A total of 19 patients were enrolled and divided into three groups: UCP $(n=9)$, FAP ( $n$ =. 7), and HUC ( $n=-3$ ) (Table 1). Overall, 37\% of patients were female and the average age was 40.6 years. There were no significant differences in baseline demographics or disease characteristics between the three groups. PDAI values were significantly higher in the UCP group $(P<0.001)$. All mucosal biopsies in the UCP group displayed histologic evidence of active pouchitis. One UCP patient received chronic ciprofloxacin therapy for the prior 8 years and one received chronic rifaximin therapy for the prior year, both with continued episodes of pouchitis. Three UCP patients had received short courses of ciprofloxacin ranging from 3 months to 1 year prior to enrollment. The remaining four UCP patients received ciprofloxacin briefly -2 years prior to enrollment. 


\section{TRFLP Analysis}

Significantly more peaks were observed in TRFLP data from mucosal samples (average total number of peaks/ sample in three digests $=64.8$, standard deviation $[\mathrm{SD}]=12.2$ ) compared to fecal samples (average total number of peaks/sample in three digests $=41.3, \mathrm{SD}=8.2$ ) with $P<0.001$ (Fig. 1). The greatest amount of similarity existed within samples from the same person, with both mucosal and fecal sample similarity greater within a subject than between subjects $(P=$ 0.027). There was no significant difference between patient groups in the degree of similarity between fecal and mucosal samples $(P>0.05)$. Between individuals, mucosal TRFLP profiles were found to be more consistent than fecal samples $(P<0.001)$.

TRFLP data from mucosal and fecal samples were significantly different between each of the three patient groups. Figure 2 displays the results of multidimensional scaling analysis comparing TRFLP data. Significant differences $(P<0.05)$ in bacterial TRFLP data were revealed between the UCP group, HUC group, and FAP group when comparing mucosal samples, fecal samples, and when both sample types were combined. Samples from the two UCP patients receiving a single long-term antibiotic were not significantly different compared to other UCP samples. Samples from the three patients who received prior short courses of antibiotics within 1 year were also not significantly different, as all nine UCP samples grouped together apart from HUC and FAP.

Approximately $15 \%$ of the total number of TRFLP peaks were matched to peak sets in the database. In an analysis of TRFLP elements that represent bacterial genera, a set of TRFs matching Lactobacillus and Streptococcus (Hae 264-5, Hpa 97-9, Alu 76, 532) were present at higher relative abundance in both mucosal and fecal samples from FAP patients compared to UCP (ratio 5:1 in mucosa, 3:1 in stool). A second set of TRFLP peaks (Hae 272-4, Hpa 222-3, Alu 440) matching Clostridium, Eubacteriurn and Roseburia genera were present at five times the 
relative abundance in stool from UCP patients compared to FAP patients. Fecal samples from HUC pouches also had fewer peaks matching Clostridium, Eubacterium, and Roseburia compared to UCP (ratio 1:15). HUC stool revealed fewer Escherichia, Streptococcus, and various sulfur-oxidizing bacteria (Hpa 496, Hae 205, Alu 74) at a ratio of 1:2 compared to UCP. Mucosal samples from HUC pouches revealed a similar smaller quantity of Escherichia, Streptococcus, and various sulfur-oxidizing bacteria compared to UCP (ratio 1:2).

[Insert Figure 1 and Figure 2]

\section{DNA Sequencing}

In all, 2304 clones were processed for each pooled sample (4608 total clones). After data processing and analysis, good quality forward and reverse reads were identified with a reliable classification at the genus level (>80\%). A total of 712 sequences were identified in the UCP pooled sample and 1015 in the FAP pooled sample.

At the phylum level, the UCP pooled sample revealed significantly more Firmicutes and Verrucomicrobia (52\% and 22\% of clones, respectively) compared to the FAP group comprised of 19\% Firmicutes and 3\% Verrucomicrobia ( $P<0.001$ and $P<0.001$, respectively). However, numbers of clones in the Bacteroidetes phylum were significantly higher in the FAP group (71\% of clones) compared to UCP (20\% of clones, $P<0.001)$. There were no significant differences between pooled samples among the Fusobacteria, Proteobacteria, and Actinobacteria phyla (Table 2). [Insert Table 2]

Multiple bacterial genera within the class Clostridia were significantly more prominent in the UCP group compared to the FAP group (Table 3). Among Clostridia, the UCP group had more clones represented by the genera Roseburia (8\% of clones in UCP versus near 0\% in FAP, $P<$ 0.001), Lachnospiraceae Incertae Sedis (28\% versus 10\%, $P<0.001)$, Clostridium (9\% versus 1\%, $P<0.001$ ), Lachnospira (2\% versus $0, P<0.001$ ), and Veillonella (3\% versus near 0\%, 
$P<0.001)$. The UCP pooled sample also had more clones from the genus Prevotella than the FAP group (6\% versus $1 \%, P<0.001)$. Finally, the UCP group revealed $22 \%$ of its pooled clones from the genus Akkermansia in the phylum Verrucomicrobia, compared to only 3\% in FAP (P < 0.001).

Members of the genus Bacteroides predominated in the pooled fecal sample of the FAP group, with 678 of 1015 (67\%) clones representing Bacteroides, compared to 99 of 712 (14\%) in UCP $(P<0.001)$. Other genera that were statistically more prominent in the FAP group were Faecalibacterium in the family Ruminococcaceae (6\% of clones in FAP, $0 \%$ in UCP, $P<$ 0.001), Parabacteroides (2\% in FAP, 0\% in UCP, $P<0.001$ ), and Escherichia (2\% in FAP, $0 \%$ in UCP, $P<0.001)$.

\section{DISCUSSION}

The results of our study suggest that the pouch microbial environment is distinctly different between patients with UC-associated pouchitis and healthy UC or FAP pouches. Using 16S ribosomal gene-based TRFLP data, we identified significant differences in bacterial communities between all three patient cohorts in both stool and mucosa. These broad differences in TRFLP profiles were further explored using DNA sequencing. Sequencing revealed multiple statistically significant variations in specific bacterial genera between pooled fecal DNA from a subgroup of patients with UCP and a subgroup of patients with FAP.

We report data that supports previously published work suggesting a dysbiosis is central to the development of pouchitis. The efficacy of both antibiotics and probiotics in ameliorating the symptoms of pouchitis underscores the theory that shifts in bacteria may be important in the generation and cessation of pouch inflammation."' However, few details are known about the precise bacterial populations in the pouch and the nature of the dysbiosis associated with pouchitis. 
Studies based on classic culturing techniques have suggested the presence of more anaerobes in pouch effluent compared to ileostomy effluent and that pouch fecal bacterial populations are similar to rectal flora. ${ }^{2022}$ A later study using similar stool culture techniques noted a greater number of facultative anaerobes in the inflamed pouch lumen compared to the noninflamed UC pouch or normal ileum. ${ }^{23}$ More recently, Duffy et a1 ${ }^{24}$ used culture-based techniques to show that sulfate-reducing bacteria were present in the stool of 8 of 10 healthy UC pouches but not in any of seven FAP pouches.

Molecular techniques have been used to define the pouch microbiota more precisely. Falk et $\mathrm{a} 1^{25}$ used TRFLP to demonstrate that pouch mucosal species in two healthy UC pouches resembled those from the colon. However, populations of Clostridium perfringens in both patients were unique to the pouch. To our knowledge, only one study, by Komanduri et al, ${ }^{\text {I I }}$ used molecular techniques to compare pouch microbiota between UC patients with and without pouchitis. Using length-heterogeneity PCR followed by sequencing, they concluded that inflamed pouch mucosa had greater species diversity and more Fusobacterium varium than healthy UC pouches or healthy deal tissue in continuity with the colon. Noninflamed UC pouches revealed more representatives from the Clostridium, enteric, and Streptococcus groups compared to pouchitis and healthy nonpouch Heal tissue. These differences between UC and health could be explained by the fact that the healthy ileal tissue was in continuity with the colon without fecal stasis. In each of these studies the healthy pouch control subjects suffered from UC, without any non-inflammatory bowel disease (IBD) pouch controls.

Our study was designed to utilize molecular techniques to identify bacterial populations unique to inflamed or healthy UC pouches compared to a non-IBD pouch, keeping pouch fecal stasis as a consistent factor across all patient groups. This design and our findings differed from the results of Komanduri et al. ${ }^{1}$ We found no increase in Fusobacterium species among inflamed 
pouches and instead noted the largest numbers of Clostridium species in the UCP group. We also noted a unique reduction in Bacteroides in the inflamed pouch compared to FAP. Our findings suggest that a mucosal and luminal dysbiosis exists in pouchitis, not only when compared to the healthy UC pouch but also when compared to a non-IBD pouch. Additionally, healthy UC pouches differed significantly from FAP pouches. This suggests an alteration in ileal pouch microbiota that may be unique to the UC disease state, with or without inflammation.

Our TRFLP results supported an overall increase in fecal Clostridium in UCP patients compared to FAP patients. This finding may be consistent with those of Falk et al. ${ }^{25}$ DNA sequencing confirmed our findings, with the genus Clostridium representing 9\% of identifiable clones in the UCP pooled sample compared to only 1\% in the pooled FAP sample. More broadly, the class Clostridia accounted for 53\% of identifiable clones in the UCP group compared to 21\% in the FAP group. The genus Eubacterium was also more prevalent in the UCP stool by TRFLP, but no known Eubacterium species were revealed by sequencing. This may be due to the difficulty of TRFLP to distinguish between Eubacterium and Clostridium species. The decrease in Lactobacillus and Streptococcus genera in UCP compared to FAP pouches also was not reflected in the sequencing data. However, this is not surprising since the number of clones identified as representing Lactobacillus and Streptococcus was less than $0.5 \%$ of the total identified. These disparities between TRFLP and sequencing results could be explained by our sampling only four patients from each group for sequencing. Importantly, TRFLP is best used to compare bacterial community structure between samples and is less precise than sequencing analysis for bacterial speciation. ${ }^{26}$

Sequencing results revealed an overall decrease of the phylum Bacteroidetes in the inflamed pouch. This finding is consistent with multiple molecular surveys of intestinal microbiota in both $\mathrm{CD}$ and $\mathrm{UC} .{ }^{27-3^{\circ}}$ Bacteroidetes play a key role in maintaining gut health, so it has been 
proposed that a relative reduction in this population may favor the development of inflammation. ${ }^{29}$ Consistent with our TRFLP data, DNA sequencing also demonstrated a significant increase in clostridia in the inflamed pouch, namely among the genera Clostridium, Lachnospiraceae, and Rosehuria. While multiple studies have shown a relative decrease in clostridia species among patients with $\mathrm{CD},{ }^{30-32}$ our findings are consistent with previous work in UC revealing more clostridia in inflamed UC rectal mucosa compared to noninflamed mucosa. ${ }^{33}$

While evidence to date supports a general shift in multiple species as being linked to the development of IBD, we note two particular genera that reflect specific theories of IBD pathogenesis. We found significantly greater numbers of the genus Roseburia among inflamed pouches compared to FAP pouches. Roseburia are flagellated commensal inhabitants of the colon. Flagellin have been shown to induce proinflammatory gene expression by activating Toll-like receptor 5 (TLR5), and patients with TLR5 polymorphisms and low levels of antiflagellin antibodies may be protected from developing CD. ${ }^{34-36}$ High levels of antiflagellin antibodies, specifically anti-CBirl antibodies to flagellin of Clostridium species, are present in 50\% of CD patients, $6 \%$ of UC patients, and may be associated with the development of pouchitis. ${ }^{37,38}$ It is unknown whether Roseburia species may play a role in TLR5 activation leading to increased mucosal inflammation. Additionally, we found the mucin-degrading genus Akkermansia of the phylum Verrucomicrobia to be significantly more prevalent in pouchitis. Several theories exist regarding the role of mucin in the protection of intestinal epithelium in IBD, with mutations, alterations, and degradation of mucins being associated with CD and UC. ${ }^{39-42}$ A recent large-scale genome-wide association study revealed that a gene encoding for mucin proteins, MUC19, was mutated significantly more often in patients with $\mathrm{CD} .{ }^{43}$ While the mucin-degrading Verrucomicrobia species and Akkermansia in particular have been identified within the human 
colon, ${ }^{44,45}$ there have been only two reports of DNA from these bacteria in patients with CD" and no previous reports of Verrucomicrobia in UC.

This study has certain limitations. TRFLP is best used to compare bacterial community structure between cohorts and is not precise in identifying species. Thus, the results of TRFLP should be interpreted as suggestive of bacterial groups, rather than specific species, and emphasis should be placed on the separation between each group. The study may have found more differences between patient groups if the sample size had been larger. Nevertheless, we were able to identify statistically significant differences in TRFLP data and clone libraries between groups. Another limitation was the pooling of DNA into groups for DNA sequencing. Individual sequencing of each sample was limited due to expense. This may have led to an apparent difference in flora between groups if only one sample had a unique bacterial profile. However, we grouped samples with similar TRFLP data in an effort to minimize the chance of one sample skewing the analysis. Additionally, the use of antibiotics in UCP patients may have altered gut flora. However, TRFLP profiles were similar in all UCP patients and distinct compared to HUC and FAP, regardless of whether the subjects had received antibiotics chronically or briefly 1-2 years before enrollment. This suggests that the dysbiosis may be unique to pouchitis and not a direct result of exposure to antibiotic medications.

In conclusion, we identified differences in the pouch microbiome between the inflamed pouch in UC, the healthy pouch in UC, and the healthy pouch in FAP. These differences in bacterial populations were evident in stool and mucosa by TRFLP analysis. 16S rDNA sequencing revealed specific differences in luminal bacterial genera, with the pouchitis group having substantially fewer Bacteroidetes and more Clostridia compared to the healthy FAP group. These findings reinforce other surveys of the micro-biome in both UC and CD. Additionally, one genus with flagellated species and one genus with mucin-degrading species were more prevalent in the 
pouchitis group, thus raising TLR5 activation and mucin degradation as possible factors in the pathogenesis of pouchitis. These results suggest that a dysbiosis may exist in both the inflamed and noninflamed UC pouch which may be central to understanding UC.

\section{REFERENCES}

1. Cheifetz A, Itzkowitz S. The diagnosis and treatment of pouchitis in inflammatory bowel disease. J Clin Gastroenterol. 2004;38:S44-50.

2. Pemberton 11-1, Kelly KA, Beart RW Jr, et al. Heal pouch-anal anastomosis for chronic ulcerative colitis. Long-term results. Ann Surg. 1.987;206:504-513.

3. Meagher AP, Farouk R, Dozois RR, et al. J ileal pouch-anal anastomosis for chronic ulcerative colitis: complications and long-term outcome in 1310 patients. Br J Surg. 1998;85:800-803.

4. Stahlberg D, Gullberg K, Liljeqvist L, et al. Pouchitis following pelvic pouch operation for ulcerative colitis. Incidence, cumulative risk, and risk factors. Dis Colon Rectum. 1996;39:1012-1018.

5. Lovegrove RE, Tilney HS, Heriot AG, et al. A comparison of adverse events and functional outcomes after restorative proctocolectomy for familial adenomatous polyposis and ulcerative colitis. Dis Colon Rectum. 2006;49:1293-1306.

6. E Mahadevan U, Sandborn WI. Diagnosis and management of pouchitis. Gastroenterology. 2003;124:1636-1650.

7. Brett PM, Yasuda N, Yiannakou JY, et al. Genetic and immunological markers in pouchitis. Eur J Gastroenterol Hepatol. 1996;8: 951-955.

8. Madden MV, McIntyre AS, Nicholls RI. Double-blind crossover trial of metronidazole versus placebo in chronic unremitting pouchitis, Dig Dis Sci. 1994;39:1193-1196. 
9. Gionchetti P, Rizzello F, Venturi A, et al. Antibiotic combination therapy in patients with chronic, treatment-resistant pouchitis. Aliment Pharmacol Ther. 1999;13:713-718,

10. Gionchetti P, Rizzello F, Helwig U, et al. Prophylaxis of pouchitis onset with probiotic therapy; a double-blind, placebo-controlled trial. Gastroenterology. 2003;124:12021209.

11, Komanduri S, Gillevet PM, Sikarnodi M, et al. Dysbiosis in pouchitis: evidence of unique microfloral patterns in pouch inflammation. Clin Gastroenterol Hepatol. 2007;5:352-360.

12. Sandborn WJ, Tremaine WJ, Batts KP, et al. Pouchitis after deal pouch-anal anastomosis: a Pouchitis Disease Activity Index. Mayo Clin Proc. 1994;69:409-415.

13. Kaplan CW, Astaire IC, Sanders ME, et al. 16S ribosomal DNA terminal restriction fragment pattern analysis of bacterial communities in feces of rats fed Lactobacillus acidophilus NCFM. Appl Environ Microbiol. 2001;67:1935-1939.

14. Chou HH, Holmes MH. DNA sequence quality trimming and vector removal. Bioinformatics. 2001;17:1093-1104.

15. DeSantis '17, Hugenholtz P, Larsen N, et al, Greengenes, a chimera-checked 16S rRNA gene database and workbench compatible with ARB. Applied Environ Microbiol. 2006;72:5069-5072.

16. Altschul SF, Gish W, Miller W, et al. Basic local alignment search tool, J Mol Biol. 1990;215;403-410.

17. Wang Q, Garrity GM, Tiedje JM, et al. Naive Bayesian classifier for rapid assignment of rRNA sequences into the new bacterial taxonomy. Applied Environ Microhiol. 2007;73:52615267.

18. Cole JR, Wang Q, Cardenas E, et al. The Ribosomal Database Project: improved alignments and new tools for rRNA analysis. Nucl Adds Res. 2008:gkn879. 
19. Gionchetti P, Rizzello F, Venturi A, et al. Oral bacteriotherapy as maintenance treatment in patients with chronic pouchitis: a double-blind, placebo-controlled trial. Gastroenterology. 2000;119:305-309.

20. Nasmyth DG, Godwin PG, Dixon MF, et al. Ileal ecology after pouch-anal anastomosis or ileostomy. A study of mucosal morphology, fecal bacteriology, fecal volatile fatty acids, and their interrelationship. Gastroenterology. 1989;96:817-824.

21. Smith FM, Coffey JC, Kell MR, et al. A characterization of anaerobic colonization and associated mucosa] adaptations in the undiseased deal pouch. Colorectal Dis. 2005;7:563570.

22. Loeschke K, Bolkert T, Kiefhaber P, et al. Bacterial overgrowth in ileal reservoirs (Koch pouch): extended functional studies. Hepatogastroenterology. 1980;27:310-316.

23. Onderdonk AB, Dvorak AM, Cisneros RL, et al, Microbiologic assessment of tissue biopsy samples from deal pouch patients. J Clin Microbiol. 1992;30:312-317.

24. Duffy M, O'Mahony L, Coffey IC, et al. Sulfate-reducing bacteria colonize pouches formed for ulcerative colitis but not for familial adenomatous polyposis. Dis Colon Rectum. 2002;45:384-388.

25. Falk A, Olsson C, Ahme S, et al. Ileal pelvic pouch microbiota from two former ulcerative colitis patients, analysed by DNA-based methods, were unstable over time and showed the presence of Clostridium perfringens. Scand T Gastroenterol. 2007;42:973985.

26, Engelbrektson AL, Korzenik JR., Sanders ME, et al. Analysis of treatment effects on the microbial ecology of the human intestine. FEMS Microbiol Ecol. 2006;57:239-250.

27. Ott SJ, Musfeldt M, Wenderoth DF, et al. Reduction in diversity of the colonic mucosa associated bacterial microflora in patients with active inflammatory bowel disease. Gut. 2004;53:685-693. 
28. Conte MP, Schippa S, Zamboni I, et al. Gut-associated bacterial microbiota in paediatric patients with inflammatory bowel disease. Gut. 2006;55:1760-1767.

29. Frank DN, St Amand AL, Feldman RA, et al. Molecular-phylogenetic characterization of microbial community imbalances in human inflammatory bowel diseases. Proc Nall Aced Sci US A. 2007;104:13780-13785.

30. Gophna U, Sommerfeld K, Gophna S, et al. Differences between tissue-associated intestinal microfloras of patients with Crohn's disease and ulcerative colitis. J Clin Microhiol. 2006;44:4136-4141.

31. Baumgart M, Dogan B, Rishniw M, et al. Culture independent analysis of ileal mucosa reveals a selective increase in invasive Escherichia coli of novel phylogeny relative to depletion of Clostridiales in Crohn's disease involving the ileum. ISME J. 2007;1:403-418.

32. Manichanh C, Rigottier-Gois L, Bonnaud E, et al. Reduced diversity of faecal microbiota in Crohn's disease revealed by a metagenomic approach. Gut. 2006;55:205-211.

33. Mylonaki M, Rayment NB, Rampton DS, et al. Molecular characterization of rectal mucosa-associated bacterial flora in inflammatory bowel disease. Inflamm Bowel Dis. 2005;11:481-487.

34. Hayashi F, Smith KD, Ozinsky A, et al. The innate immune response to bacterial flagellin is mediated by Toll-like receptor 5. Nature. 2001; 410:1099-1103.

35. Gewirtz AT, Navas TA, Lyons S, et al. Cutting edge: bacterial flagellin activates basolaterally expressed TLR5 to induce epithelial proinflammatory gene expression. .1 Immunol. 2001;167:1882-1885.

36. Gewirtz AT, Vijay-Kumar M, Brant SR, et al. Dominant-negative TLR5 polymorphism reduces adaptive immune response to flagellin and negatively associates with Crohn's disease. Am J Physiol Ga.strointest Liver Physiol. 2006;290:G1157-1163. 
37. Targan SR, Landers CJ, Yang H, et al. Antibodies to CBirl flagellin define a unique response that is associated independently with complicated Crohn's disease. Gastroenterology. 2005;128:2020-2028.

38. Fleshner P, Ippoliti A, Dubinsky M, et al. Both preoperative perinuclear antineutrophil cytoplasmic antibody and anti-CBirl expression in ulcerative colitis patients influence pouchitis development after Heal pouch-anal anastomosis. Clin Gastroenterol Hepatol. 2008;6:561-568,

39. Einerhand AW, Renes IB, Makkink MK, et al. Role of mucins in inflammatory bowel disease: important lessons from experimental models. Eur J Gastroenterol Hepatol. 2002;14:757-765.

40. Kyo K Parkes M, Takei Y, et al. Association of ulcerative colitis with rare VNTR alleles of the human intestinal mucin gene, MUC3. Hum Mol Genet. 1999;8:307-311.

41. Parker N, Tsai HH, Ryder SD, et al. Increased rate of sialylation of colonic mucin by cultured ulcerative colitis mucosal explants. Digestion. 1995;56:52-56.

42. Tytgat KM, van der Wal JW, Einerhand AW, et al. Quantitative analysis of MUC2 synthesis in ulcerative colitis. Biochem Biophys Res Commun. 1996;224:397-405.

43. Barrett JC, Hansoul S, Nicolae DL, et al. Genome-wide association defines more than 30 distinct susceptibility loci for Crohn's disease. Nat Genet. 2008;40:955-962.

44. Collado MC, Derrien M, Isolauri E, at al. Intestinal integrity and Akkermansia muciniphila, a mucin-degrading member of the intestinal microbiota present in infants, adults, and the elderly. App! Environ Microhiol. 2007;73 :7767-7770.

45. Derrien M, Collado MC, Ben-Amor K, et al. The mucin degrader Akkermansia muciniphila is an abundant resident of the human intestinal tract. App! Environ Microhiol. 2008;74:1646-1648. 
46. Bibiloni R, Mangold M, Madsen KL, et al. The bacteriology of biopsies differs between newly diagnosed, untreated, Crohn's disease and ulcerative colitis patients. J Med Microbiol. 2006;55:1141-1149.

TABLE 1. Patient Demographic and Disease Characteristics

\begin{tabular}{lcccr} 
& UCP $(n=9)$ & FAP $(n=7)$ & HUC $(n=3)$ & P-value \\
\hline Mean age in years (range) & $32.2(22-61)$ & $45.5(29-55)$ & $39.3(26-55)$ & 0.15 \\
Gender & $\mathrm{M}=2(22 \%)$ & $\mathrm{M}=4(57 \%)$ & $\mathrm{M}=1(33 \%)$ & 0.48 \\
& $\mathrm{~F}=7(78 \%)$ & $\mathrm{F}=3(43 \%)$ & $\mathrm{F}=2(67 \%)$ & 0.51 \\
Mean duration of pouch in years & $5.9(1.5-13)$ & $8.7(1.4-21)$ & $12.3(2-20)$ & 0.31 \\
Mean duration of UC in years & $8.8(2.5-14)$ & $\mathrm{n} / \mathrm{a}$ & $14.7(3-25)$ & $<0.001^{*}$ \\
Mean PDAI score (range) & $8.8(7-12)$ & 0 & $1(1)$ & \\
*Statistically significant. UCP, UC familial adenomatous & HUC, healthy & & \\
\hline
\end{tabular}

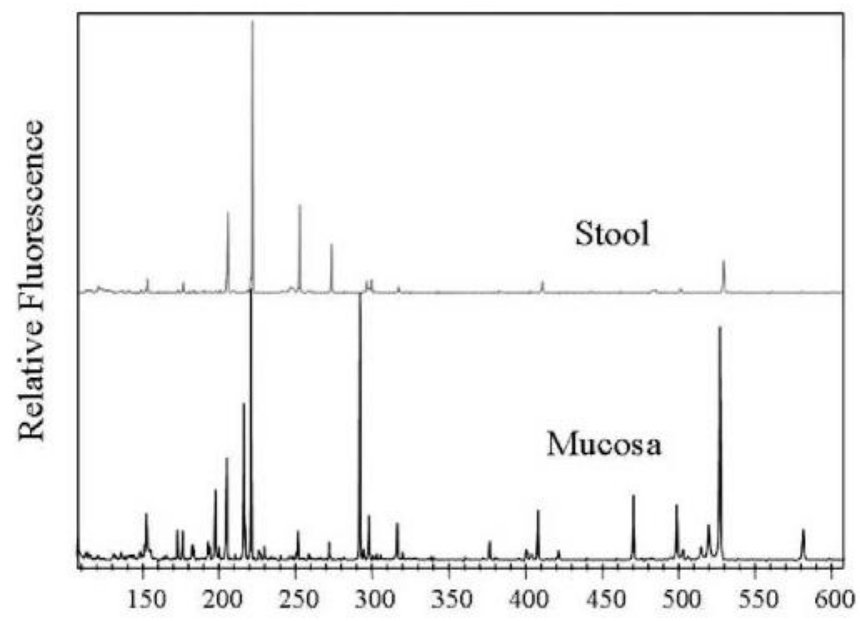

TRF length (nucleotides)

FIGURE 1. Example of raw TRFLP data. TRFLP data (Hpa digest) from one patient (stool and mucosal samples) illustrating the difference in diversity made apparent by TRFLP. Each peak represents one or more taxonomic unit(s) of bacteria defined by the single TRF length. Note the increased number of peaks in the mucosal sample. 

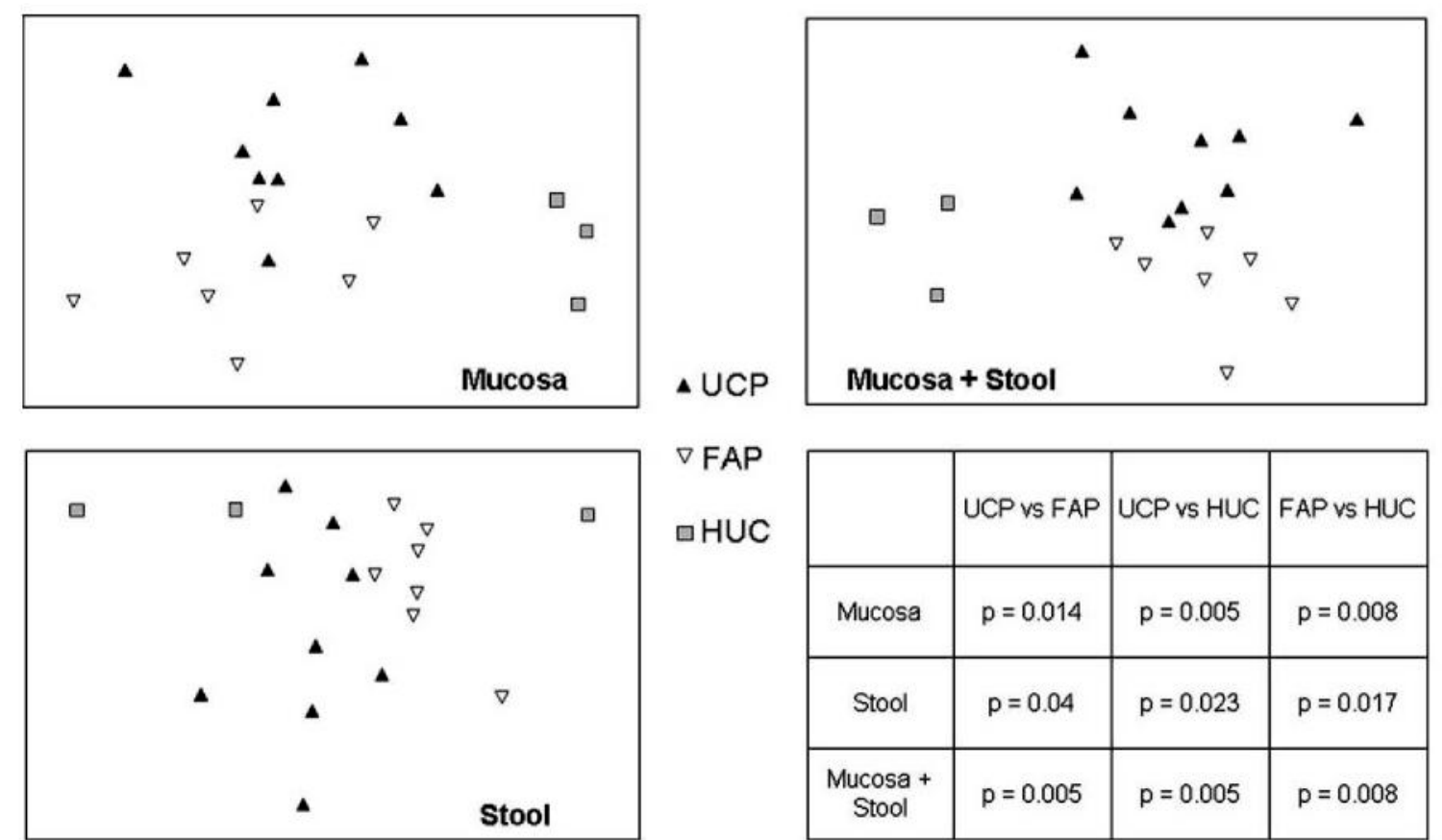

\begin{tabular}{|c|c|c|c|c|}
\hline $\begin{array}{l}\nabla \text { FAP } \\
\square \text { HUC }\end{array}$ & & UCP vs FAP & UCP vs HUC & FAP vs HUC \\
\hline & Mucosa & $p=0.014$ & $p=0.005$ & $p=0.008$ \\
\hline & Stool & $p=0.04$ & $p=0.023$ & $p=0.017$ \\
\hline & $\begin{array}{c}\text { Mucosa + } \\
\text { Stool }\end{array}$ & $p=0.005$ & $p=0.005$ & $p=0.008$ \\
\hline
\end{tabular}

FIGURE 2. Multidimensional scaling (MDS) analysis of TRFLP data. MDS analysis compares complex profiles, here TRFLP peaks, in two-dimensional space with the most similar profiles being closest spatially and those most dissimilar being farthest apart. Each shape represents one patient's TRFLP profile. Differences between groups were evaluated using ANOSIM. P-values for each comparison are listed.

TABLE 2. Comparison of DNA Sequencing Results by Phylum

Percent of Identifiable DNA Clones (Absolute Number)

\begin{tabular}{lccc}
\cline { 2 - 4 } & UCP & FAP & $P$-value \\
\hline Bacteroidetes & $20 \%(144)$ & $71 \%(716)$ & $<0.001$ \\
Firmicutes & $52 \%(379)$ & $19 \%(216)$ & $<0.001$ \\
Fusobacteria & $1 \%(4)$ & $<1 \%(4)$ & $\mathrm{NS}$ \\
Proteobacteria & $5 \%(30)$ & $5 \%(44)$ & $\mathrm{NS}$ \\
Verrucomicrobia & $22 \%(155)$ & $3 \%(35)$ & $<0.001$ \\
Actinobacteria & $<1 \%(0)$ & $<1 \%(1)$ & $\mathrm{NS}$ \\
\hline
\end{tabular}

NS, not significant; UCP, UC pouchitis; FAP, familial adenomatous polyposis. 


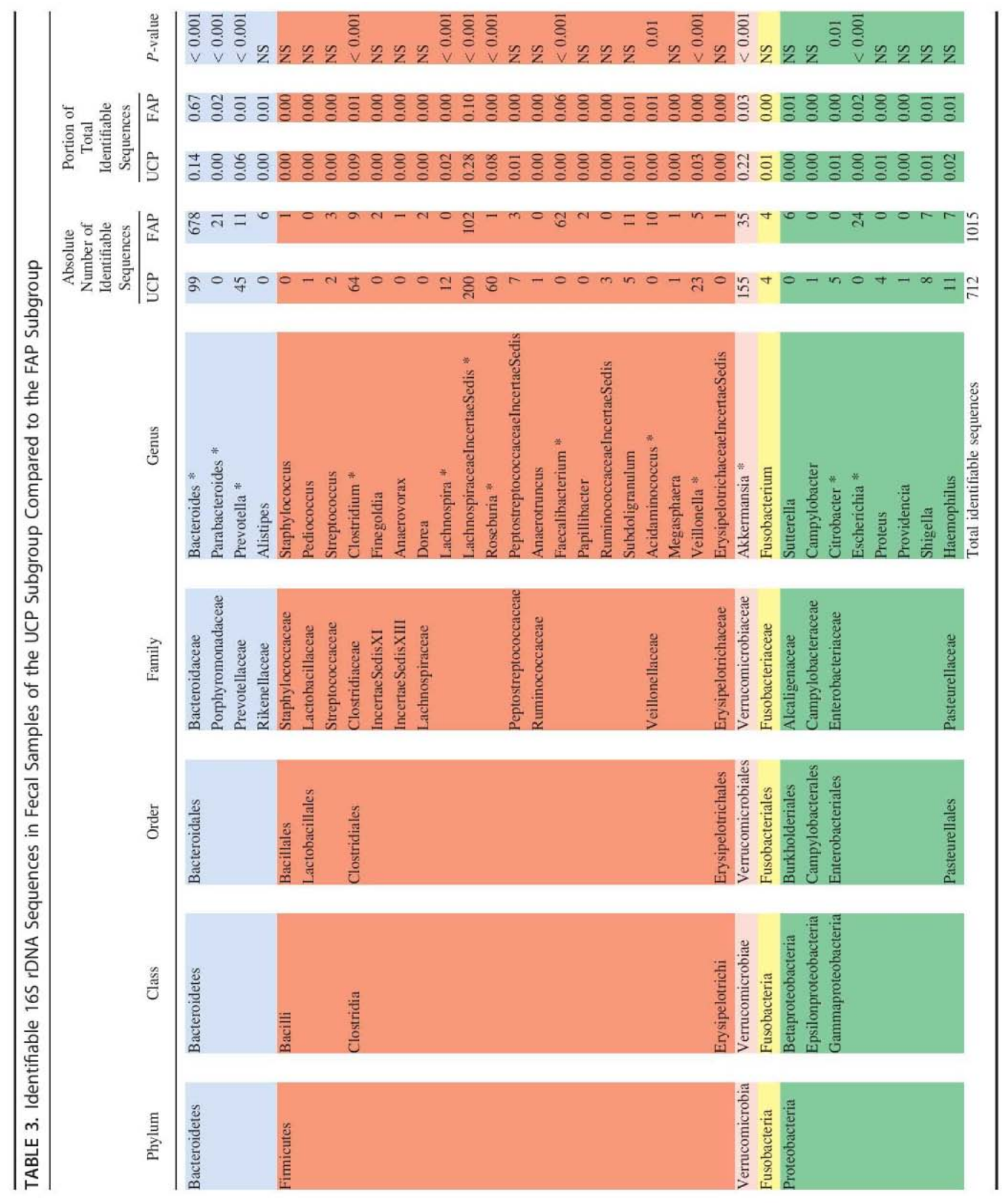

